\title{
ON A SEQUENCE TRANSFORMATION WITH INTEGRAL COEFFICIENTS FOR EULER'S CONSTANT
}

\author{
C. ELSNER
}

(Communicated by Andrew Bruckner)

ABSTRACT. Let $\gamma$ denote Euler's constant, and let

$$
s_{n}=\left(1+\frac{1}{2}+\cdots+\frac{1}{n-1}\right)-\log n \quad(n \geq 2) .
$$

We prove by Ser's formula for the remainder $\gamma-s_{n}$ that for all integers $n \geq 1$ and $\tau \geq 2$ there are integers $\mu_{n, 0}, \mu_{n, 1}, \ldots, \mu_{n, n}$ such that $\mu_{n, 0} s_{\tau}+\mu_{n, 1} s_{\tau+1}+\cdots+\mu_{n, n} s_{\tau+n}=\gamma+O_{\tau}\left((n(n+1)(n+2) \cdot \cdots \cdot(n+\tau))^{-1}\right)$, where the constant in $O_{\tau}$ depends only on $\tau$.

The coefficients $\mu_{n, k}$ are explicitly given and are bounded by $2^{3 n+\tau-1}$.

By $\gamma$ we denote Euler's constant; it is well known that the sequence $\left(s_{n}\right)_{n \geq 0}$ defined by

$$
s_{n}=\left(1+\frac{1}{2}+\cdots+\frac{1}{n-1}\right)-\log n \quad(n \geq 2)
$$

tends to $\gamma$, where

$$
s_{n}=\gamma+O\left(n^{-1}\right) \quad(n \geq 2) .
$$

J. Ser [6] has proved that the remainder of $\gamma-s_{n} \quad(n \geq 2)$ can be expressed as an infinite sum with rational terms: Let

$$
t_{m+2}=-\frac{1}{(m+1) !} \int_{0}^{1}(0-x)(1-x) \cdots(m-x) d x \quad(m \geq 0) .
$$

Then

$$
\gamma=\frac{1}{n} \sum_{m=0}^{\infty} \frac{t_{m+2}}{\left(\begin{array}{c}
m+n \\
m
\end{array}\right)}+\left(1+\frac{1}{2}+\cdots+\frac{1}{n-1}\right)-\log n \quad(n \geq 2) .
$$

(See also [3, pp. 14-15].)

But, of course, $\gamma-s_{n}$ can be written in a lot of different ways. For example, we get by Euler's summation formula for any positive integers $n \geq 2$ and $k$ :

$$
\gamma=s_{n}+\frac{1}{2 n}+\sum_{j=1}^{k} \frac{B_{2 j}}{2 j \cdot n^{2 j}}+R(n, k),
$$

Received by the editors August 5, 1993.

1991 Mathematics Subject Classification. Primary 65B05; Secondary 40A05. 
where $B_{m}$ are the Bernoulli numbers and

$$
|R(n, k)| \leq \frac{4}{n} \sqrt{\frac{k}{\pi}}\left(\frac{k}{\pi e n}\right)^{2 k}
$$

(see [4]).

$A$ historical remark. The representation of $\gamma$ by the right-hand side of (2) was the main tool in P. Appell's attempt to prove the irrationality of $\gamma$ in 1926 [1]. Appell himself quickly discovered his error and within a week he published a retraction. An outline of this incorrect proof is sketched in [2]. In what follows we apply a linear sequence transformation to the class of those sequences, where the error term can be expressed by a sum like (2). First we introduce some notation:

$$
\begin{gathered}
(\alpha)_{m}=\alpha(\alpha+1)(\alpha+2) \cdots(\alpha+m-1), \quad(\alpha)_{0}=1 \quad\left(\alpha \in \mathbb{R}, \quad m \in \mathbb{Z}_{>0}\right) ; \\
\mu_{n, k}(\tau)=\mu_{n, k}=(-1)^{n+k} \frac{(\tau+k)_{n}}{n !}\left(\begin{array}{l}
n \\
k
\end{array}\right) \quad\left(n \in \mathbb{Z}_{\geq 0}, 0 \leq k \leq n\right),
\end{gathered}
$$

where $\tau \in \mathbb{Z}_{>0}$ is fixed. Note that $\mu_{n, k} \in \mathbb{Z} \quad\left(n \in \mathbb{Z}_{\geq 0}, 0 \leq k \leq n\right)$.

Theorem 1. Let $\left(v_{n}\right)_{n \geq 0}$ be a sequence of real numbers such that

$$
\begin{gathered}
\lim _{n \rightarrow \infty} v_{n}=s, \\
v_{n}=s-\sum_{m=1}^{\infty} \frac{c_{m}}{(n+\tau)_{m}} \quad(n \geq 0),
\end{gathered}
$$

where $\left(c_{m}\right)_{m \geq 1}$ denotes a sequence of real numbers satisfying

$$
0 \leq c_{m} \leq C \cdot(m+\sigma) ! \quad(m \geq \max \{1 ;-\sigma\})
$$

for some constant $C>0$ and some

$$
\sigma \in \mathbb{Z} \quad \text { with } \sigma<\tau-2 \text {. }
$$

Then we have for

$$
\begin{gathered}
e_{n}=\left(\sum_{k=0}^{n} \mu_{n, k} v_{k}\right)-s: \\
\left|e_{n}\right| \leq C \cdot \frac{(n+\sigma+1) ! \cdot(\tau-\sigma-3) !}{(n+\tau-\sigma-2) !} \quad(n \geq \max \{0 ;-(\sigma+1)\}) .
\end{gathered}
$$

The linear sequence transformation given in (6) belongs to a certain class of so-called nonregular methods; a general theory of such transformations can be found in [7] (see Chapter 2.3.5).

Theorem 2. For $n \geq 1$ and $\tau \geq 2$ we have

$$
\left|\sum_{k=0}^{n} \mu_{n, k} s_{k+\tau}-\gamma\right| \leq \frac{(\tau-1) !}{2 n(n+1)(n+2) \cdots \cdots(n+\tau)} \text {. }
$$

From this theorem we get a very good approximation to $\gamma$ in terms of $s_{n}, s_{n+1}, \ldots, s_{2 n}$ by choosing $\tau=n \geq 2$ :

$$
\left|\sum_{k=0}^{n} \mu_{n, k} s_{n+k}-\gamma\right| \leq \frac{1}{2 n^{2}\left(\begin{array}{c}
2 n \\
n
\end{array}\right)} \leq n^{-3 / 2} \cdot 4^{-n} \text {. }
$$


There are linear sequence transformations for $\left(s_{n}\right)_{n \geq 0}$ with nonintegral coefficients, which converge more rapidly to $\gamma$ than the transformation given in Theorem 2 (see [5]). But from an arithmetical point of view in number theory it is much more attractive to accelerate the convergence by transformations with integral coefficients.

Proof of the theorems. From $\sum_{k=0}^{n} \mu_{n, k}=1$ we have by (3) and (6) for every $n \geq 0$ :

$$
\begin{aligned}
e_{n} & =-\sum_{k=0}^{n} \sum_{m=1}^{\infty}(-1)^{n+k} \frac{(k+\tau)_{n}}{k ! \cdot(n-k) ! \cdot(k+\tau)_{m}} c_{m} \\
& =-\sum_{m=1}^{\infty} c_{m} \sum_{k=0}^{n}(-1)^{n+k} \frac{(k+n+\tau-1) !}{k ! \cdot(n-k) ! \cdot(k+m+\tau-1) !} .
\end{aligned}
$$

From $c_{m} \geq 0$ in (4) we conclude that the infinite series $\sum_{m=1}^{\infty} \frac{c_{m}}{(n+\tau)_{m}} \quad(n \geq 0)$ converges absolutely, and so we may interchange the sums in (7). We express the terms in (8) again by Pochhammer's symbol; this gives for $n \geq 0$ :

$$
\begin{aligned}
e_{n}= & (-1)^{n+1} \cdot \frac{(n+\tau-1) !}{n !} \sum_{m=1}^{\infty} \frac{c_{m}}{(m+\tau-1) !} \sum_{k=0}^{n} \frac{(n+\tau)_{k} \cdot(-n)_{k}}{k ! \cdot(m+\tau)_{k}} \\
= & (-1)^{n+1} \cdot \frac{(n+\tau-1) !}{n !} \\
& \cdot\left(\left(\sum_{m=1}^{n}+\sum_{m=n+1}^{\infty}\right) \frac{c_{m}}{(m+\tau-1) !} \sum_{k=0}^{\infty} \frac{(n+\tau)_{k} \cdot(-n)_{k}}{k ! \cdot(m+\tau)_{k}}\right)
\end{aligned}
$$

(since $(-n)_{k}=0$ if $\left.k>n\right)$. Let $a, b, c$ be real numbers, $c \neq 0,-1,-2, \ldots$;

$$
F(a, b ; c ; x)=\sum_{k=0}^{\infty} \frac{(a)_{k} \cdot(b)_{k}}{k ! \cdot(c)_{k}} x^{k} .
$$

We only treat the case $c-a-b>0$; for this it is well known that

$$
F(a, b ; c ; 1)= \begin{cases}\frac{\Gamma(c) \cdot \Gamma(c-a-b)}{\Gamma(c-a) \cdot \Gamma(c-b)} & \text { if } c-a, c-b \neq 0,-1,-2, \ldots \\ 0 & \text { otherwise }\end{cases}
$$

The sum on the right-hand side of (10) occurs in (9) with

$$
a=n+\tau, \quad b=-n, \quad c=m+\tau .
$$

From $m \geq 1$ in (9) we have $m+\tau>\tau$, hence $c>a+b$. Note $c-a \leq 0 \Leftrightarrow$ $m \leq n$. By (11) we now see that $e_{n}$ equals

$$
\begin{gathered}
(-1)^{n+1} \frac{(n+\tau-1) !}{n !} \sum_{m=n+1}^{\infty} \frac{c_{m}}{(m+\tau-1) !} \frac{(m+\tau-1) ! \cdot(m-1) !}{(m-n-1) ! \cdot(m+n+\tau-1) !} \\
=(-1)^{n+1} \frac{(n+\tau-1) !}{n !} \sum_{m=0}^{\infty} c_{m+n+1} \frac{(m+n) !}{m ! \cdot(m+2 n+\tau) !} \quad(n \geq 0) .
\end{gathered}
$$

Now let

$$
n_{0}=\max \{0 ;-(\sigma+1)\}
$$


$n \geq n_{0}$ implies $m+n+1 \geq n+1 \geq \max \{1,-\sigma\}$. Thus for $n \geq n_{0}$ we estimate $e_{n}$ from (12) by (4),

$$
\left|e_{n}\right| \leq C \cdot \frac{(n+\tau-1) !}{n !} \sum_{m=0}^{\infty} \frac{(m+n+\sigma+1) ! \cdot(m+n) !}{m ! \cdot(m+2 n+\tau) !} \quad\left(n \geq n_{0}\right) .
$$

We treat the infinite sum in (13) in the same way as we did with the inner sum in (8). For $n \geq n_{0}$ we get

$$
\left|e_{n}\right| \leq C \cdot \frac{(n+\tau-1) ! \cdot(n+\sigma+1) !}{(2 n+\tau) !} \sum_{m=0}^{\infty} \frac{(n+\sigma+2)_{m} \cdot(n+1)_{m}}{m ! \cdot(2 n+\tau+1)_{m}} .
$$

To apply (11) again we now define in (10):

$$
a=n+\sigma+2, \quad b=n+1, \quad c=2 n+\tau+1 .
$$

From (5) we have $2 n+\tau+1>2 n+\sigma+3$, hence $c>a+b$. That gives

$$
\begin{aligned}
\left|e_{n}\right| & \leq C \cdot \frac{(n+\tau-1) ! \cdot(n+\sigma+1) !}{(2 n+\tau) !} \frac{\Gamma(2 n+\tau+1) \cdot \Gamma(\tau-\sigma-2)}{\Gamma(n+\tau-\sigma-1) \cdot \Gamma(n+\tau)} \\
& =C \cdot \frac{(n+\sigma+1) ! \cdot(\tau-\sigma-3) !}{(n+\tau-\sigma-2) !} \quad\left(n \geq n_{0}\right) .
\end{aligned}
$$

This proves the theorem.

Theorem 2 follows immediately from Theorem 1 and (2): Put

$$
c_{m}=-\frac{1}{m} \int_{0}^{1}(0-x)(1-x) \cdots(m-1-x) d x \quad(m \geq 1) .
$$

Hence

$$
t_{m+1}=\frac{1}{(m-1) !} \cdot c_{m} \quad(m \geq 1) ;
$$

from the definition of $s_{n}$ and (2) we get $^{1}$

$$
\begin{aligned}
s_{n+\tau} & =\gamma-\frac{1}{n+\tau} \sum_{m=1}^{\infty} \frac{t_{m+1}}{\left(\begin{array}{c}
m+n+\tau-1 \\
m-1
\end{array}\right)}=\gamma-\sum_{m=1}^{\infty} c_{m} \cdot \frac{(n+\tau-1) !}{(m+n+\tau-1) !} \\
& =\gamma-\sum_{m=1}^{\infty} \frac{c_{m}}{(n+\tau)_{m}} \quad(n \geq 0) .
\end{aligned}
$$

This is (3), where $\tau \geq \mathbb{Z}_{\geq 2}$. We get an integer $\sigma$ from (15) by

$$
0 \leq c_{m} \leq \frac{1}{m} \int_{0}^{1} x \cdot(m-1) ! d x=\frac{(m-1) !}{2 m} \leq \frac{(m-2) !}{2} \quad(m \geq 2) .
$$

Hence we may choose $\sigma=-2, C=\frac{1}{2}, n_{0}=1$; and (5) holds.

Theorem 2 now follows from Theorem 1 , where $v_{n}=s_{n+\tau}$.

At last note that

$$
\begin{aligned}
\mu_{n, k} & =(-1)^{n+k} \frac{(\tau+k)_{n}}{n !}\left(\begin{array}{l}
n \\
k
\end{array}\right)=(-1)^{n+k}\left(\begin{array}{c}
n+k+\tau-1 \\
n
\end{array}\right)\left(\begin{array}{l}
n \\
k
\end{array}\right) \\
& =(-1)^{n+k}\left(\begin{array}{c}
n+k+\tau-1 \\
n-k, k, k+\tau-1
\end{array}\right) \quad(n \geq 1,0 \leq k \leq n)
\end{aligned}
$$

${ }^{1}$ Note that (2) holds for $s_{n+\tau}$ with $n \geq 0$ and $\tau \geq 2$. 
and

$$
\left|\mu_{n, k}\right| \leq 2^{n+k+\tau-1} \cdot 2^{n} \leq 2^{3 n+\tau-1}
$$

\section{ACKNOWLEDGMENT}

I express my thanks to Professor G. Mühlbach for encouragement and his valuable help.

\section{REFERENCES}

1. P. Appell, Sur la nature arithmétique de la constante d'Euler, C. R. Acad. Sci. I Math. 15 (1926), 897-899.

2. R. G. Ayoub, Partial triumph or total failure, Math. Intelligencer 7 (1985), 55-58.

3. L. B. W. Jolley, Summation of series, second edition, Dover, New York, 1961.

4. D. E. Knuth, Euler's constant to 1271 places, Math. Comp. 16 (1962), 275-280.

5. I. M. Longman, Increasing the convergence rate of series, Appl. Math. Comput. 24 (1987), 77-89.

6. J. Ser, L'intermediaire des mathematiciens, Gauthier-Villars, Paris, Ser. 2, 1925.

7. J. Wimp, Sequence transformations and their applications, Academic Press, New York, 1981.

Institut für Mathematik, Universität Hannover, Postfach 60 09, 30060 HanNover, GERMANY

E-mail address: elsner@math.uni-hannover.de 